\title{
Die geomorphologisch-bodengeographische Deutung der pleistozänen Sedimente und der fossilen Böden an der Playa de Barañán (westl. La Coruña) und bei Cangas de Foz (Nord-Galizien)
}

\author{
KARL-UlRich Brosche *)
}

\begin{abstract}
Coastal pit section, coastal catena, coastal terrace, interpretation, paleosol, solifluction, eolian deposit, horizon differentiation, Würmian, C 14 dating.

North Galicia, Playa de Barañán
\end{abstract}

$\mathrm{Ku} \mathrm{rzf}$ assung: Aus dem nördlichen Galizien (NW-Spanien) werden von zwei Lokalitäten (Playa de Barañán, westl. La Coruña, und Cangas de Foz, westl. Ribadeo) jungpleistozäne Sediment-Boden-Folgen beschrieben, die auf einem 3-5 m ü. M. gelegenen Felssockel bzw. auf einer ca. $25 \mathrm{~m}$ ï. $\mathrm{M}$. gelegenen Küstenterrasse auflagern. Mehrere ${ }^{14} \mathrm{C}$-Daten sowie Vergleiche mit früher aufgenommenen Sediment-Boden-Folgen in der näheren und weiteren Umgebung lassen den Schluß zu, daß in beiden Fällen eine nahezu vollständige würmzeitliche Schichtenfolge entwickelt ist, die von fossilen Braunerden bzw. kräftigen fossilen $\mathrm{A}_{\mathrm{h}}$-Horizonten gegliedert ist. Zwischen die fossilen Böden bzw. Bodensedimente sind möglicherweise äolisch entstandene Schluffe bzw. Sande und Solifluktionsschuttdecken eingeschaltet. Die wahrscheinlich altwürmzeitlichen Profilteile zeichnen sich durch viel Holzkohle aus und weisen auf eine holzreiche Vegetation hin.

[Geological and Pedological Interpretation of the Pleistocene Sediments and Fossil Soils at the Playa de Barañán (W of La Coruña) and near Cangas de Foz (N-Galicia) ]

A b s t r a c t : Upper Pleistocene sediment-soil profiles were investigated from a rock basement at 3-5 metres a.s.l. and a coastal terrace at 25 metres a.s.l. at two sites in northern Galicia/north-west Spain (Playa de Barañán, west of La Coruña, and Cangas de Foz, west of -Ribadeo). Several ${ }^{14} \mathrm{C}$-dates and comparisons with previously investigated sediment-soil profiles from the same area indicate that in both cases an almost complete Würmian stratification exists, ranging from fossil brown soils to thick fossil $A_{h}$-horizons. Possibly wind-blown silts or sands and solitluctional rock-fragments are embedded between the fossil soils and soil sediments. Sections of the profile, probably Lower Würmian in age, contain a considerable quantity of charcoal and indicate a wood-rich vegetation.

\section{Einleitung: Arbeitsgebiet, Problemstellung und Literaturüberblick}

Westlich der Provinzhauptstadt La Coruña liegt an der Playa de Barañán bei Campo Branqueza (westl. des Dorfes Arteijo) das erste im folgenden zu behandelnde Profil direkt am Meer. Auf einem niedrigen Felssockel treten steil zum Meer abfallende Sedimente auf, in die mehrere fossile Böden eingeschaltet sind. Dieses Profil wurde von Franz (1967:35 ff.) ausführlich beschrieben und gedeutet, während NoNN $(1966,1969)$ es nur sehr kurz im Rahmen seiner Gesamtübersicht erwähnt.

Das zweite Profil westlich von Foz im westlichen Asturien wurde auf einer ca. $25 \mathrm{~m}$ ü. M. gelegenen Meeresterrasse im Jahre 1977 von mir neu entdeckt. Es zeichnet sich durch eine mehrfach wiederkehrende Serie von fossilen Böden und Sedimenten aus. Auf-

*) Anschrift des Autors: Priv.-Doz. Dr. K.-U. B ros ch e, Institut für Physische Geographie der Freien Universität Berlin, Grunewaldstraße 35, 1000 Berln 41.

Dem Fachbereich Geowissenschaften der FU Berlin danke ich für die finanzielle Unterstützung bei einer Reise im Jahre 1977. Herrn Prof. Dr. Mook, Groningen, bin ich für die Datierung mehrerer Proben in seinem Labor zu Dank verpflichtet, Herrn Prof. Dr. K. Kaiser, Berlin, für die Vermittlung einer finanziellen Hilfe für die Probendatierungen. 
grund seines Gehalts an organischem Material bietet es sich für eine Datierung und Analyse an.

Die dargestellten Befunde sind Ergänzungen zu jüngst veröffentlichten Untersuchungen zu Sediment- und Bodensequenzen von der westgalizischen Küste (BROSCHE 1982), die recht genau gedeutet und datiert werden konnten. Sie sind aber auch als Fortsetzung und Ergänzung früherer bodengeographisch-landschaftsökologischer Untersuchungen zu betrachten, die auf der Iberischen Halbinsel im Anschluß an RutTe (1960) von RoHDENburg \& Sabelberg (1969a, b, 1973), Franz (1967), Brunnacker \& Ložek (1969), Brosche \& WALTHER (1977) sowie Brosche (1978) durchgeführt worden sind und eine detaillierte geomorphologisch-bodengeographische Analyse der Paläoböden und Paläosedimente zum Inhalt haben. Im Gegensatz zu den meisten früheren Untersuchungen kann bei dieser ebenso wie bei Brosche (1982) auf mehrere ${ }^{14} \mathrm{C}-\mathrm{D}$ aten zurückgegriffen werden.

\section{Das Küstenprofil an der Playa de Barañán bei Campo Branqueza (westlich La Coruña)}

\subsection{Das Profil und seine Beschreibung}

Das Profil an der Playa de Barañán bei Campo Branqueza (Abb.1) wird bereits kurz bei NoNN (1966:349) erwähnt und zusammen mit anderen Profilen als Beispiel für ein Ouljien-Niveau angeführt. Franz (1967: 35-38) behandelt dieses Profil dagegen ausführlich. Er betont, daß es sich hier zusammen mit dem Küstenprofil bei Mougas in Westgalizien (vgl. auch Brosche 1982) um das vollständigste Küstenprofil in Galizien handelt.

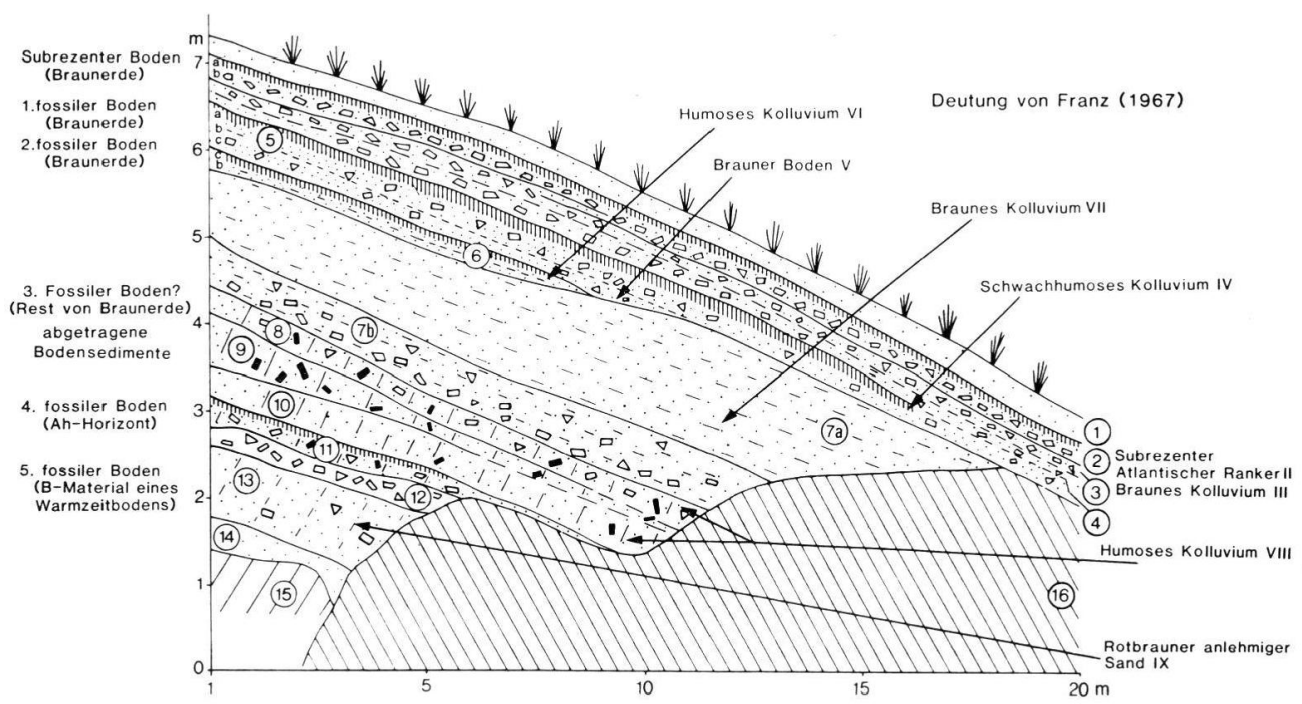

Abb. 1: Sediment-Boden-Abfolge in der Playa de Barañán bei Campo Branqueza (westl. La Coruña). Schräganschnitt, deshalb z. T. etwas generalisiert. Oben Häufung von drei subrezenten bzw. fossilen $\mathrm{A}_{\mathbf{h}}$-Horizonten, unten mehrfach Holzkohlehäufung. 
Das Profil (Abb. 1) liegt im Ostteil der Playa de Barañán unterhalb eines in Betrieb befindlichen Steinbruchs am östlichen Ortsrand der Streusiedlung Campo Branqueza unterhalb der Landstraße Arteijo-Cayón. Es handelt sich hier ohne Zweifel um die gleiche Lokalität, die Franz (1967: 35-36) in seinem Profil I beschreibt und deutet. $\mathrm{Zu}$ den folgenden Ausführungen sind die Abb. 1, deren rechte untere Ecke etwa 2-3 m ü. M. liegt, und die Figuren 1-3 (Tafel 1) heranzuziehen. ${ }^{1}$ )

Auf der linken Seite der Abb. 1 sind die von mir erkannten fossilen Böden und Bodensedimente eingetragen. Auf der rechten Seite derselben Abbildung sind die von FrANZ (1967: 35-37) als fossile Böden oder Sedimente angesprochenen Schichten mit den bei FranZ zur findenden römischen Zahlen eingezeichnet. Das Profil liegt an einem $15^{\circ}-20^{\circ}$ abfallenden Hang, der in der Abb. 1 schräg angeschnitten ist. Im östlichen Teil an der Basis ist ein unebener Felssockel aus Amphibolit sichtbar, der bis $3-5 \mathrm{~m}$ ü. M. hinaufreicht.

Tab. 1: Die Schichtenfolge nach meinen Geländeaufnahmen im Sommer 1977

Schicht Mächtigkeit Geologisch-pedologische Beschreibung

Schichtenfolge bei Franz (1967)

\footnotetext{
1

$20-40 \mathrm{~cm}$ rezenter bis subrezenter, weißgrauer Flugsand, schwach humos, vorwiegend Grobsand, vor allem mit Strandgras bewachsen

$20 \mathrm{~cm}$ schwarzer subrezenter $\mathrm{A}_{\mathrm{h}}-\mathrm{Horizont}$ auf Feinsand mit Schuttbesatz, krümelnd, dicht, Farbe 10 YR $2 / 2$

$10 \mathrm{~cm}$ wie $2 \mathrm{a}$, nur dunkelbraun und schwächer humos

$20-60 \mathrm{~cm}$ brauner $\mathrm{B}_{\mathrm{v}}$-Horizont der subrezenten Braunerde; oben dunkelbraun $(20 \mathrm{~cm})$, unten heller braun (in trockenem Zustand gelb bis braungelb), wenige Steine in sandigschluffiger Grundmasse

$30-40 \mathrm{~cm}$ braunes, schwach humoses, sandig-schluffiges Material mit wenigen Schuttstücken

$20 \mathrm{~cm}$ schwarzbraune humose Schicht, 1 . fossiler $\mathrm{A}_{\mathrm{h}}$ - Hor iz o n t, Grundmasse sandig-schluffig, mit Schutt durchsetzt, ${ }^{14} \mathrm{C}$-Alter des Alkali-Extraktes: $19160 \pm 220 \mathrm{BP}$ (GrN-9446); ${ }^{14} \mathrm{C}-$ Alter des humosen organischen Materials: $16330 \pm 100 \mathrm{BP}(\mathrm{GrN}-8325)$

$5 b$

$$
\left.\begin{array}{c}
10-20 \mathrm{~cm} \text { mittelbraun, } \\
\text { sonst wie } 5 \mathrm{a} \\
10-20 \mathrm{~cm} \text { hell-braun, } \\
\text { sonst wie } 5 \mathrm{a}
\end{array}\right\} \quad \begin{gathered}
\text { schwacher } \\
\mathrm{B}_{\mathrm{v}} \text {-Horizont }
\end{gathered}
$$

$20 \mathrm{~cm}$ humoser, mittelbrauner, schluffiger Sand mit vielen Steinen, 2. fossiler $A_{h}-H o r i z o n t$, eventuell etwas gekappt

1) Alle Sedimente wurden nur makromorphologisch aufgenommen und nicht im Labor analysiert, was den Wert mancher bodengenetischer Aussagen einschränkt. Trotz dieses Mangels scheint mir die erstmalige Beschreibung, Deutung und Datierung (Cangas de Foz) bzw. die neuerliche Behandlung des Kliffprofils an der Playa de Barañán sinnvoll zu sein, da durch dessen Vergleich mit dem Profil von Tapia de Casariego und unter Heranziehung mehrerer neuer ${ }^{14} \mathrm{C}-\mathrm{Daten}$ eine Reihe von neuen Ergebnissen erzielt werden kann.
}

(1)


Schicht Mächtigkeit Geologisch-pedologische Beschreibung

Schichtenfolge bei FranZ (1967)

$6 \mathrm{~F}$

$7 a$

$7 \mathrm{~b}$

8
$10-20 \mathrm{~cm}$ brauner schluffiger Sand (schwach entwickelter $\mathrm{B}_{\mathrm{v}}-$ Horizont?)

1,0-1,2 $\mathrm{m}$ brauner (schwach rötlicher?), s t e in $\mathrm{fr}$ e i e r, lehmigsandiger Schluff (7,5 YR 4/4)

VII

0,5 m lehmiger, sandiger Schluff, z. T. locker, ohne Holzkohle mit wenigen Steinen, (Farbe 7,5 YR 4/4), an Basis Steinsohle aus grobem Schutt (Abtragungsdiskordanz)

30-40 cm brauner sandiger Lehm mit Holzkohle (Rest eines fossilen Bodens?), mittelbraun wie 6a, fest, wenige Steine enthaltend

$50 \mathrm{~cm}$ mit Holzkohle durchsetzter, sehr fester sandiger Lehm, schwarz-dunkelbraun, mit braunen Schmitzen (Bodenmaterial?) durchsetzt, offenbar verlagertes Sediment, unten mehr Holzkohle als oben, Profil abwärts auskeilend

$30 \mathrm{~cm}$ dunkelbrauner, fester lehmiger Sand, etwas Holzkohle enthaltend

40-50 cm tief schwarzbrauner, mit Schutt und Holzkohle durchsetzter, lehmig-sandiger Schluff (als 3. fossile r $\mathrm{A}_{\mathrm{h}}$ - Horizon t gedeutet)

$20 \mathrm{~cm}$ Schuttband mit wenig feinem Matrixmaterial

$1,2 \mathrm{~m}$ schwach rotbrauner (Farbe: 7,5 YR 5/6), anlehmiger Sand mit wenigen Steinen (Interglazialboden?)

IX

$20-40 \mathrm{~cm}$ fossiler hellgelber Flugsand

Kristallines Gestein, Gneis oder Amphibolit

Franz (1967: 35-37) charakterisiert die Schichtenfolge dagegen folgendermaßen:

\section{Profil 1}

Playa Barañán bei Campo Branqueza an der carretera local von Arteijo nach Cayón ${ }^{2}$ )

I.

$0-20 \mathrm{~cm} \mathrm{Rezenter}$ Flugs a nd, schwächst humoser, loser Sand, vorwiegend Grobsand, mit Strandgras und anderen Strandpflanzen bewachsen. Farbe $10 \mathrm{YR}$ 4/2. Er ist an den Hängen bis mindestens $20 \mathrm{~m}$ über den Meeresspiegel emporgeweht und sitzt scharf auf

II.

$20-40 \mathrm{~cm} \mathrm{Subrezenter}$ A t lantischer Ranker, stark humoser Feinsand mit schwachem Kiesbesatz, schwach krümelnd, ziemlich dicht lagernd, Farbe 10 YR 2/2 übergehend in

III.

$40-90 \mathrm{~cm}$ Braunes Kolluvium mit vielen eckigen Steinen bis zu 10-20 cm Durchmesser, ganz überwiegend kristallines Material (mehr oder weniger verwitterter Granit und Gneis), aber ganz vereinzelt auch gerundete Quarze, eingelagert in Feinerde: anlehmiger Sand, dicht lagernd, oben dunkel braun (7 1/2 YR 3/2), die unteren $40 \mathrm{~cm}$ heller (7 1/2 YR 4/4) rasch übergehend in

$\left.{ }^{2}\right)$ Druckfehler wurden von mir verbessert. Bei Franz (1967) in Kursivdruck erscheinende Worte wurden hier ges perrt. 
IV. $\quad 90-105 \mathrm{~cm} \mathrm{Schwach} \mathrm{hum} \mathrm{oses} \mathrm{Kolluvium,} \mathrm{viel} \mathrm{heller} \mathrm{grau} \mathrm{als} \mathrm{Hori-}$ zont II und Horizont VI (Farbe nicht mit Farbtafel bestimmt, weil Horizont in der Aufschlußwand nicht zugänglich), starke Anreicherung eckiger, mindestens faustgroßer Steine, petrographisch wie anstehendes Kristallin. Dieser Horizont keilt nach Westen aus, wo die Horizonte am Rande des Sandstrandes zum Meeresspiegel absinken. Er geht nach unten über in

V. 105-155 cm Brauner Boden, anlehmiger feiner Sand, verfestigt, in den höheren Partien reich an eckigen Steinen, in den tieferen stellenweise vollkommen steinfrei, an der Basis dunkler gefärbt. Farbe hier 7 1/2 YR $4 / 4$, übergehend in

VI. $\quad 155-180 \mathrm{~cm}$ Humoses Kolluvium mit sehr vielen faust- bis kopfgroßen Steinen, die petrographisch dem anstehenden Kristallin entsprechen, Feinmaterial humoser anlehmiger Sand, Farbe 7 1/2 YR 3/2, rasch übergehend in

VII. $\quad 180-270 \mathrm{~cm}$ Braunes Kolluvium, schwach lehmiger Feinsand, dicht gelagert mit reichlichem Steinbesatz, dieser aber doch geringer als in Horizont VI und die Steine im Durchschnitt kleiner, Farbe $71 / 2$ YR 4/4, stellenweise dunkler (5 YR 3/4-4/4) allmählich übergehend in

VIII. $\quad 270-330 \mathrm{~cm}$ Humoses Kolluvium, wieder reicher an eckigen überwiegend faust- bis kopfgroßen Steinen, der Feinerdeanteil ist stark humos, sehr schwach lehmiger Sand, dieser etwas gröber als in Horizont VII, Farbe 5 YR $2 / 2$, einzelne kleine braune Flecken sind vorhanden (Gleyflecken) ebenso einzelne Holzkohlenstückchen, überwiegend in

IX. 330-430 (450) cm Rotbrauner anlehmiger Sand, dicht lagernd und leicht verfestigt, relativ wenige eckige Steine enthaltend, diese bis über faustgroß. Farbe oben 5 YR 3/4-4/4 unten heller. Ubergehend in

X. 400-450 (500) cm Grober heller Flugsand, etwas verfestigt, aber sehr leicht zerreiblich, steinfrei. In der Aufschlußwand befinden sich in diesem Horizont zahlreiche Nistlöcher von Erdbienen. Material vollendet gerundet, viel runder als der rezente Dünensand. Farbe feucht 7 1/2 YR 5/7, trocken etwa 10 YR 6/5. Wird vom anstehenden Fels unterlagert, reicht aber stellenweise bis zum Sandstrand herab und wird dort vom rezenten, viel helleren Sand, der in Form einer kleinen Düne angeweht ist, überdeckt.

XI. $\quad 450(500) \mathrm{cm}$ u. tiefer: Anstehendes Kristallin (vorwiegend Amphibolit).

Die Schichtenbeschreibungen von Franz (1967) und mir lassen sich gut in Einklang bringen. Lediglich im Bereich des Horizontes VIII bei Franz (1967:37) wurden von mir weitere Differenzierungen vorgenommen, was möglicherweise an den jetzt günstigeren Aufschlußverhältnissen liegt. Bezüglich der Deutung der Sedimente und Böden als humose und braune Kolluvialmassen, als braune Böden und als Atlantischer Ranker durch Franz (1967) sind jedoch Umdeutungen angebracht.

\subsection{Deutung und Datierung des Profils}

Meine Deutung dieser Sediment- und Bodenserie ist auf der linken Seite der Abb. 1 dargestellt (s. o.). Bei der jetzt folgenden Deutung kommt mir die Kenntnis des Profils von Tapia de Casariego (BRosche 1978: 161-168; BRosche \& Walther 1977:313-319) zu Hilfe, von dem zwei ${ }^{14} \mathrm{C}-$ Daten vorliegen. Es stimmt in den Grundzügen mit dem Profil an der Playa de Barañán überein. Unter dem rezenten bis subrezenten Flugsand liegt eine erste subrezente Braunerde mit den Horizonten $A_{h}$ und $B_{v}$ (Schichten $2 a$ und $2 b$ 
in Tab. 1). M. E. ist die Ausgliederung eines „subrezenten Atlantischen Rankers“ (Franz 1967:36) ohne $B_{V}$-Horizont als Unterboden unrichtig. Das „Braune Kolluvium“ von FRANZ bildet nämlich den $\mathrm{B}_{\mathrm{v}}$-Horizont der subrezenten Braunerde.

Als fossile Bodenbildung (Schichten $5 \mathrm{a}, 5 \mathrm{~b}, 5 \mathrm{c}$ in Tab. 1) tritt eine zweite Braunerde mit den Horizonten $A_{h}$ und $B_{v}$ auf. Der $A_{h}$-Horizont wurde mit $16330 \pm 100 \mathrm{BP}$ (GrN-8325) datiert. ${ }^{3}$ ) In diesem Fall gliederte FraNZ (1967:36) ein „schwach humoses Kolluvium" (Horizont IV) und einen „Braunen Boden“ (Horizont V) gesondert aus. Als vorläufige zeitliche Einstufung dieser Bodenbildung kommt das Bölling-Interstadial in Frage. Aber auch das Stillfried-B-Interstadial kommt als Bildungszeit in Betracht, sofern man eine Kontamination durch Sickerwässer am Hang unterstellt, was zu einer "Verjüngung" der ${ }^{14} \mathrm{C}$-Alter führt. Diese zeitliche Einstufung erscheint mir jedoch wegen der unten dargestellten Parallelisierungsmöglichkeiten mit dem Profil von Tapia de Casariego eher unwahrscheinlich.

Die Schichten $6 a$ und $6 \mathrm{~b}$ (Tab. 1, Abb. 1) sind als zweite fossile Bodenbildung, und zwar als 2. fossile Braunerde (besser: als Braunerde-Ranker), anzusprechen. Franz (1967: 35 f.) spricht statt dessen von einem „Humosen Kolluvium“ (Horizont VI). Dieser recht schwach entwickelte fossile Boden hat am Hang möglicherweise eine schwache oberflächliche Kappung bzw. eine geringfügige Verlagerung in seinen oberen Partien erfahren.

Franz (1967) fand die 1,2 m mächtige, fast steinlose braune schluffige Schicht 7a und die Schicht 7b in Tab. 1 und Abb. 1 (Horizont VII bei Franz) offenbar nicht in der steinlosen Fazies wie ich vor. Er bezeichnet (S.36) diesen mit Steinen durchsetzten Abschnitt des Profils als „Braunes Kolluvium“. Ich schließe nicht aus, daß in der Schicht 7a ein überwiegend äolisch entstandenes Sedimentpaket vorliegt, das, da sich keine Steine finden ließen, in einer Phase fehlender oder schwacher fluvialer und solifluidaler Aktivität am Hange entstanden ist. Ein ähnliches, farblich dem Löß vergleichbares Sediment trafen wir auch im Profil von Tapia de Casariego im oberen Profilabschnitt an (BRosche \& WALther 1977: 317, Schicht 1). Dort wird es wie hier von einem stark steinhaltigen, schluffig-sandigen Feinsediment (dort: Schicht 1a) unterlagert, das der Schicht 7b (Profil Playa de Barañán) entsprechen könnte. Bei Tapia de Casariego folgt unter diesem zweigliedrigen Sedimentkomplex ein brauner Interstadialboden (BROsCHE 1978: 165; BROSCHE \& Walther 1977: 315, 317). Holzkohledatierungen ergaben ein Alter von 23700 Jahren vor heute oder älter. Unter dem zweigliedrigen Komplex tritt im Profil an der Playa de Barañán als Schicht 8 ebenso wie in Tapia de Casariego ein brauner fester, sandiger Lehm auf, der auch Holzkohle enthält. Es ist wahrscheinlich, daß die beschriebenen Schichtfolgen von Tapia de Casariego und von der Playa de Barañán miteinander korrelieren. Weitere Gemeinsamkeiten, die dies bestätigen, treten im unteren Teil des Profils auf (s. u.). Auf der linken Seite der Abb. 1 wurde der braune feste sandige Lehm, die Schicht 8, als möglicher fossiler B-Horizont eines Bodens eingestuft, dessen Oberboden heute fehlt. ${ }^{4}$ )

Die Schichten 8-11 des Profils an der Playa de Barañán faßt Franz (1967:37) als humoses Kolluvium (bei ihm Horizont VIII) zusammen. Innerhalb dieser Serie fallen die dunklen, z. T. tiefschwarzen oder braunschwarzen Farben, das gehäufte Vorkommen von Holzkohle, die braunschwarze Fleckung (von FrANz 1967: 37, als Pseudovergleyung gedeutet) sowie die Steine auf. Die Steinanreicherung ist über der Schicht 13 (Horizont IX

$\left.{ }^{3}\right)$ Der Alkali-Extrakt derselben Probe ergab ein ${ }^{14} \mathrm{C}$-Alter von $19160 \pm 220 \mathrm{BP}(\mathrm{GrN}-9446)$. Als zu datierendes Materil diente eine ausreichende Menge stark humosen Materials.

$\left.{ }^{4}\right)$ Es sei hier darauf hingewiesen, daß auch in Mitteleuropa in Lößprofilen an dieser Stelle in den Würmprofilen eine kräftige Diskordanz bzw. eine Abtragungsphase nachweisbar ist, durch die der Lohner Boden entweder ganz beseitigt oder stark aufgearbeitet wurde (vgl. z. B. SEMMEL 1968; Rohdenburg 1968; Brosche \& Walther 1977, 1980). 
bei FraNZ), einem rotbraunen anlehmigen Sand, den FRANZ (1967:49,50) als Interglazialboden des Eem oder älter deutet ${ }^{5}$ ), so stark, daß sie auf eine kräftige Umlagerungszone hinweist.

Vor der stratigraphischen Einordnung der Schichten 9-12 (Abb. 1) erfolgt ihre Deutung. Die Schicht 9, die durch ihre schwarzen, dunkelbraun gefleckten sandigen Lehme und Holzkohlestückchen gekennzeichnet ist, deute ich als verlagertes und schwach pseudovergleytes Bodenmaterial, das möglicherweise aus einem fossilen Ranker mit kräftigem $A_{h}$-Horizont hervorging. Die kräftig entwickelte schwarze Schicht 11 deute ich als $A_{h}$ Horizont eines fossilen Rankers.

Bezüglich der Deutung der rötlich-braunen Schicht 13 als Interglazialboden gibt es m. E. keine Alternative. Die liegenden äolischen gelben Flugsande (Schicht 14) und der Felssockel aus Gneis bzw. Amphibolit stützen diese Deutung. Ich stufe diesen rötlichbraunen Interglazialboden (Schicht 13) als Eem-Boden ein, während Franz (1967: 49-51) eher an ein älteres Interglazial zu denken scheint. Die liegenden gelben Sande (Schicht 14) deute ich mit Franz (1967) als Flugsande bzw. als ältere Dünensande, die in Meeresnähe ausgeblasen wurden. Die einzelnen Körner sind nach Franz (1967:37) sehr stark abgerollt und bestehen vorwiegend aus Quarz, daneben aus Erzen. Sehr dünne limonitische Überzüge bedingen die leichte Verfestigung des Sandes, wobei die Limonithäutchen amorph sind. Daß sich in dem Felssockel (Schicht 15) wahrscheinlich ein fossiler Abrasionssockel verbirgt, ergibt sich daraus, daß FRANZ $50 \mathrm{~m}$ weiter westlich in einem Profil (das dem hier behandelten nach FRANz [S. 38] völlig entspricht) unter dem fossilen Flugsand völlig gerundete Quarzschotter als Strandgerölle fand.

Die Deutung der Ablagerungsbedingungen des gelben Flugsandes über dem Amphibolitsockel durch Franz (1967: 49) muß allerdings korrigiert werden. Das Zitat bei Franz (S. 49), der das Profil Cayón nennt und damit nur das Profil von der Playa de Barañán (zwischen Arteijo und Cayón) meinen kann, da es in seinem Aufsatz kein Profil Cayón gibt, lautet: „7) Der gelbe Flugsand, der die braunen Böden des Küstenprofils von Cayón unterlagert, bezeugt, daß vor der Bildung dieser Böden eine Periode intensiver Erosion mit gleichzeitig relativ hohem Meeresstand nach bereits erfolgter Absenkung des Festlandes auf das heutige Niveau bestanden hat. "Nach dem heutigen Kenntnisstand deute ich den gelben Flugsand über dem Felssockel knapp über dem heutigen Meeresspiegel nicht durch die Annahme „einer Periode intensiver Erosion...., sondern als äolische Sedimente, die am Ende der Rißkaltzeit, als der Meeresspiegel noch unter dem heutigen lag und im Begriff war, auf das Ouliien-Niveau einige Meter uiber dem heutigen Meeresspiegel anzusteigen, aus den Sandflächen zwischen damaligem Meeresspiegel und heutigem Festland in Richtung auf das heutige Festland ausgeblasen worden sind. Eine Periode intensiver Erosion, die Franz vielleicht, ohne es zu sagen, aus den großen Strandgeröllen $50 \mathrm{~m}$ westlich des Profils ableitet, ist nicht zu erschließen. Vielmehr lassen sich die großen Gerölle zwischen gelbem Flugsand und Anstehendem auch hier an der Playa de Barañán als marin bewegte und deshalb so gut gerundete Strandgerölle deuten, die nach der Flugsandphase im Zuge des Meeresspiegelanstiegs im Eeminterglazial abgelagert worden sind.

In diesem Zusammenhang sei auch daran erinnert, daß NoNN (1966:349) OuljienStrände aus diesem Gebiet erwähnt, ohne aber die auflagernde Sediment- und Bodenfolge ausführlich zu beschreiben und ohne genaue Ortsangaben zu machen. Erwähnt sei ferner, daß Franz (1967: 38) im Südteil der gleichen Playa etliche Meter von der Küste entfernt in einem $6 \mathrm{~m}$ tiefen Brunnenschacht über dem anstehenden Kristallin wieder die gelben Flugsande (Schicht 14 in Abb. 1) antraf, so daß man mit einer weitflächigen Unterlagerung der würmzeitlichen Sedimente durch alte Flugsande rechnen muß (ich selbst fand diese Feinsedimente auch am westlichen Rand der Playa in einer kleinen Bucht). Der alte Ouljien-Strand liegt hier nach allen bisher vorliegenden Befunden nur ein bis einige (ca. 2-3) Meter über dem heutigen Meeresspiegel.

5) Diese Einstufung ergibt sich für Franz aufgrund des Umstandes, daß er (1967: 49) den braunen Böden in den oberen Teilen der Profile ein letztinterglaziales, allenfalls ein würminterstadiales Alter zuschreibt. 
Wichtig scheint mir ferner der Hinweis zu sein, daß im Profil von Tapia de Casariego im Bereich zwischen dem Eem-Interglazialboden (der dort als farbintensiver, stark verlehmter Pseudogley vorliegt) und dem dort ${ }^{14} \mathrm{C}$-datierten Mittelwürm-Interstadialboden eine Sedimentfolge liegt, die sich ebenfalls durch schwarze und braunschwarze Farben, durch viel Holzkohlebeimengungen, durch Anzeichen von Pseudovergleyung und durch Schutteinlagerungen auszeichnet (BRosche \& Walther 1977: 317). Im Profil von Tapia de Casariego konnte ein nahezu autochthoner kalkloser $A_{h}$-Horizont mit einer Bleichzone darunter und außerdem Reste eines zweiten fossilen $A_{h}$ Horizontes gefunden werden. Somit ergibt sich eine bemerkenswerte Ubereinstimmung dieses Profilteils in Tapia de Casariego mit dem entsprechenden Profilteil an der Playa de Barañán.

Dieser Profilabschnitt (Abb. 1: Schichten 9-12) wird deshalb wie in Tapia de Casariego, wo am ca. $26^{\circ}$ geneigten Hang an einem Dellenrand noch zusätzlich zwei Bleichzonen unter den genannten $A_{h}$-Horizonten auftreten, als Altwürm-Abschnitt gedeutet. In dieses Bild, das bereits früher bei der Deutung des Profils von Tapia de Casariego unter genauer Kenntnis der Würmlöß-Abfolgen in Mitteleuropa gezeichnet wurde, paßt überraschend gut der bereits erwähnte Befund, daß über dem Eem-Interstadialboden eine kräftige Umlagerungszone liegt (Schicht 12).

Es ist mir an einem zweiten Beispiel gelungen, wesentliche Grundzüge der PaläoBodenentwicklung, wie sie in Mitteleuropa am Beispiel der Würmlöß-Stratigraphie (Schönhals, Rohdenburg \& Semmel 1964; Fink 1956; Brunnacker 1957; Rohdenburg \& Meyer 1966; Rohdenburg 1971; Semmel 1968, 1974; Ruske \& WÜNSche 1961; Unger \& RAU 1964; LIEBEROTH 1959-1962 u. a.) gewonnen wurden, zwanglos in nordspanischen Küstenprofilen mit Schluffbeteiligung wiederzufinden.

Noch eine weitere Ubereinstimmung mit dem Profil von Tapia de Casariego sei angefügt: An der Playa de Barañán (Tafel 1; Fig. 3, rechter Teil der Abb. 1)wie dort (Brosche \& WALTHER 1977, Fig. 1) laufen die jüngsten Schichten in Richtung nach $\mathrm{N}$ auf das Meer zu zusammen und lassen sich nicht mehr differenzieren.

Bezüglich der Deutung der Sedimente und Böden im Profil in der Playa de Barañán bei Campo Branqueza durch Franz (1967:35-38) sei abschließend folgendes hervorgehoben:

1. Die von Franz $(1967: 36,37)$ herausgestellten Kolluvialmassen sind nicht als Kolluvialmassen, sondern, wie Fig. 2 und besonders Fig. 3 deutlich zeigen, als Solifluktionsschuttdecken mit einer klaren Einregelung der Längsachsen im Sinne von POSER \& Hövermann (1951) zu deuten. Ähnliche Schuttdecken wie die in Fig. 3 sichtbare ziehen in ganz Galizien bei ausreichender Hangneigung bis an das Meer hinab, wie NoNN (1966), Tricart (1966) und Brosche (1978) gezeigt haben. Eine Schichtung dieser Schuttmassen, die auf fluviale (kolluviale) Prozesse schließen läßt, ist nicht sichtbar.

2. Durch diese Deutung der Hangschuttdecken als Solifluktionsschuttdecken ist entgegen der Annahme von Franz (1967:50 und 51) der Nachweis von eiszeitlichen Bodenfrosterscheinungen für Nordwestspanien erbracht. Zu diesem Ergebnis kommen nicht nur spanische Autoren (u. a. Llopís Lladó 1955, 1957; Llopís Lladó \& JoRda 1957, Hernández Pachéco, Jorda Cerda \& Martinez 1957) sondern auch Tricart (1966) und NonN (1966: 86, Planche II u. Fig. 18), der Kryoturbationserscheinungen bei Guittiriz (Nationalstraße Lugo-Lo Coruña) beschreibt und abbildet (vgl. auch BROSCHE 1978: 154-175).

3. Durch die eiszeitlichen Solifluktionsprozesse wurden die in Phasen eines günstigeren Klimas gebildeten Böden (Braunerden, Braunerderanker mit kräftigem $\mathrm{A}_{\mathrm{h}}$-Horizont) z. T. aufgearbeitet und hangab verlagert.

4. Die humosen und braunen Kolluvialmassen i. S. von FRANZ (1967) sind nicht das Ergebnis von Pluvialphasen (Franz 1967:51) auf der Iberischen Halbinsel, sondern von feucht-kalten Abschnitten innerhalb eines Glazials. 
5. Die Ausgliederung eines besonderen Bodentyps durch FRANZ (1967:49, 50), nämlich eines Atlantischen Rankers („AC-Boden“), der nur aus einem humosen Horizont $A_{h}$ und einem C-Horizont bestehen soll, ist für die Lokalitäten, die Franz (1967) anführt, ungerechtfertigt. Hierbei handelt es sich vielmehr um kräftige $\mathrm{A}_{\mathrm{h}}$-Horizonte von Braunerden oder Braunerderankern.

6. Böden, die nur aus einem kräftigen $A_{h}$-Horizont bestehen, lassen sich in Galizien an sehr steilen Hängen über silikatischem Ausgangsgestein finden (vgl. BroscHe 1982: 73). An steilen Hängen hat Franz keine Bodenprofile untersucht.

7. Die Ausgliederung von eigenständigen fossilen braunen Böden durch FRANZ (1967: 36 und 49-51) ist nicht haltbar; es handelt sich dabei vielmehr um $B_{v}$-Horizonte von Braunerden, die meistens zusammen mit ihrem humosen Oberboden ( $A_{h}$-Horizont) autochthon vorkommen.

Die zeitliche Einstufung der Schichten des Profils als jung-, mittel-, altwürmzeitliche und eeminterglaziale Sedimente sowie Böden entspricht den Vorstellungen über das eiszeitliche Verhalten des Meeresspiegels: die würmglazialen Schuttdecken- und Bodenserien fallen hier - wie in Tapia de Casariego - steil ab und tauchten möglicherweise bis in nicht bekannte Tiefen unter den heutigen Meeresspiegel ab. Dieses Verhalten entspricht der bekannten Tatsache, daß der würmglaziale Meeresspiegel tiefer lag als der heutige. Die unter dem Eem-Boden liegenden alten gelben Flugsande sind dagegen wie die schluffig-sandigen Sedimente, auf denen der Eem-Boden entwickelt ist, aufgrund ihrer Höhe von 3-5 $\mathrm{m}$ ü. M. und ihrer flächenhaften Ausdehnung als interglaziale oder altrißzeitliche Sedimente anzusehen. Sie wurden wahrscheinlich ïberwiegend während eines Meerestiefstandes in Strandnähe abgelagert. Aber auch ein früheemzeitliches Alter ist möglich, wie sich nach dem aktualistischen Prinzip aus der rezenten/subrezenten Flugsandbildung (Schicht 1 in Abb. 1) ablesen läßt.

Die gesamte Sediment- und Bodenfolge an der Playa de Barañán läßt auf einen etwa fünffachen (vielleicht sechsfachen) Wechsel von starker morphologischer Aktivität, schwächerer morphologischer Aktivität und Stabilität schließen. Starke morphologische Aktivität heißt: Ablagerung von Solifluktionsschuttdecken, schwächere morphologische Aktivität heißt: Ablagerung der steinfreien und fast steinfreien lößfarbigen, sandigschluffigen Sedimente $=$ Schichten $7 \mathrm{a}, 7 \mathrm{~b}$, eventuell durch äolische und schwache fluviale Prozesse. Die morphologische Stabilität äußerte sich in der Bildung von Rankern mi kräftigen $\mathrm{A}_{\mathrm{h}}$-Horizonten und Braunerden in klimatisch günstigeren, wärmeren, vegetationsreicheren Phasen der Bodenbildung. Für einen kürzeren Abschnitt des Würmglazials (für Teile des Hochglazials und für das Spätglazial) wurde dies bereits von Nonn (1966: 289) durch Interpretation des Pollenprofils bei Mougas abgeleitet.

\section{Das Profil bei Cangas de Foz (Nordostgalizien)}

\subsection{Beschreibung von drei Einzelprofilen}

Das Profil in der Nähe von Cangas de Foz liegt zwischen Vivero und Cangas de Foz am Kilometerstein 416 der Nationalstraße C 642 El Ferrol del Gaudillo-Ribadeo. Der Aufschluß ist ca. $7 \mathrm{~km}$ nordwestl. von Foz gelegen und befindet sich in einer großen, frisch ausgehobenen Baugrube auf einer ca. $25 \mathrm{~m}$ ü. M. gelegenen Küstenterrasse (Abb. 2). Ca. 200-300 m nördlich des großen Aufschlusses ist an der heutigen Küste ein fossiler Strand aufgeschlossen, der in schräg einfallenden Schiefern angelegt und von einer sandigkiesigen Deckschicht überlagert wird. (vgl. oberes Foto auf Planche XXVI bei NonN 1966, neben S. 436). 


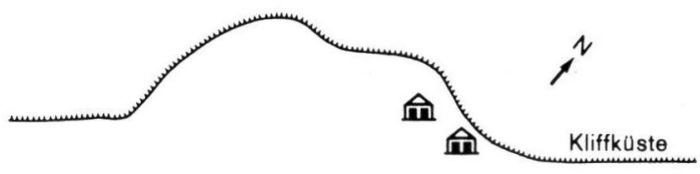

Schmalspurbahn El Ferrol-Ribadeo
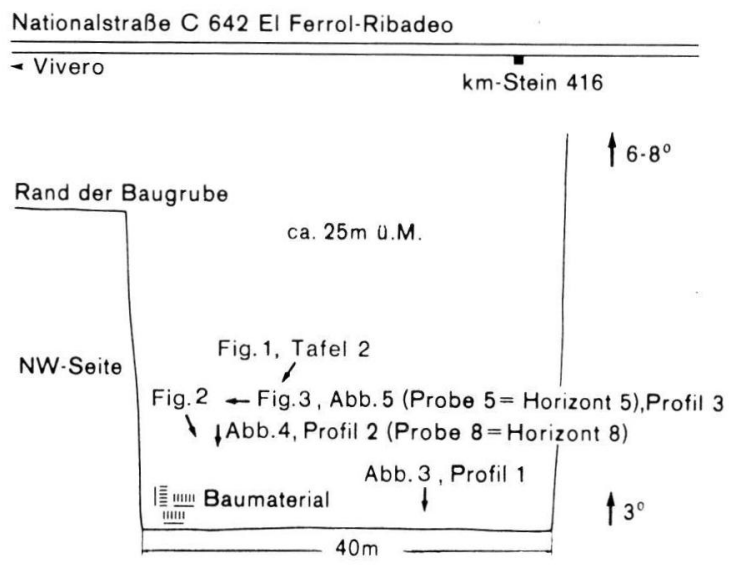

Abb. 2: Übersichtsskizze des Aufschlusses bei Cangas de Foz am Kilometerstein 416 der Nationalstraße C 642 von El Ferrol nach Ribadeo, ca. $7 \mathrm{~km}$ nordwestl. von Foz. Baugrubengrund für große Halle auf einer ca. $25 \mathrm{~m}$ ü. M. gelegenen Küstenterrasse. Lage aller Abbildungen u. Figuren sichtbar.

Abb. 2 gibt den Grundriß der Grube mit den Lokalitäten wieder, an denen die Abb. 3-5 und die Fig. 1-3 (Tafel 2) entstanden sind. Bei den aufgeschlossenen Sedimenten, die in über $6 \mathrm{~m}$ Mächtigkeit auf einer Küstenterrasse liegen, handelt es sich um eine mehrfache Abfolge von überwiegend sandigen (z. T. schwach schluffigen) lößfarbigen Feinsedimenten und von Sandsteinschuttdecken mit einer sandigen Matrix. In diese Sedimente sind neben einem kräftigen basalen humosen Horizont zwei durchgehende kräftige fossile $A_{h}$-Horizonte eingeschaltet, während lokal noch zwei weitere humose Schichten auftreten, deren Deutung problematisch ist.

Wie der rechte Teil von Fig. 1 (Tafel 2) zeigt, fallen die fossilen $A_{h}$-Horizonte von $\mathrm{SE}$ nach NW flach in Richtung auf die Küste $\mathrm{ab}$. Sie folgen damit etwa der Oberfläche, die ebenfalls - zunächst mit $3{ }^{\circ}$, weiter meerwärts mit $6^{\circ}-8^{\circ}-$ abfällt. Wie Fig. 1 (Tafel 2) weiter erkennen läßt (links hinter den Baumaterialien), kleiden die fossilen humosen Horizonte eine flache Delle aus, die an der heutigen Oberfläche nicht mehr erkennbar ist, in früherer Zeit aber aktiv gewesen sein muß. In der Tiefenlinie dieser Delle, die auch in Fig. 2 (Tafel 2) wieder gut sichtbar wird, kam es zum Teil zur Bildung recht mächtiger humoser Horizonte, z. T. aber auch zu geringfügigen Erosionserscheinungen und fluvialen Verschwemmungen von Material. Um das weite Spektrum der wechselnden Aufschlußverhältnisse möglichts zu erfassen, werden drei Profile (Abb. 3-5) exemplarisch behandelt. Sie seien im folgenden einzeln beschrieben und gedeutet, bevor einige allgemeine bodengeographische Schlußfolgerungen gezogen werden. 


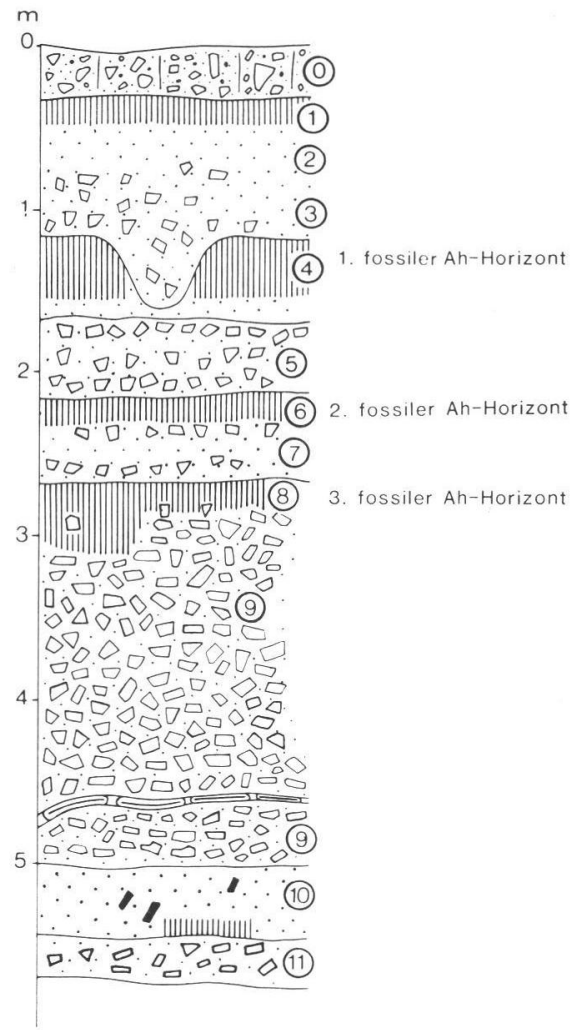

Abb. 3: SW-NE-Wand mit Profil 1. Zur Lage vergleiche Abb. 2. Numerierung entspricht der Numerierung der Schicht-Nummern im Text.

Tab. 2: Profil 1, SW-NE-Wand des Profils Cangas de Foz (vgl. Abb. 3)

Schicht Mächtigkeit Geologisch-bodengeographische Ansprache

0

1

2

3

4

$35 \mathrm{~cm}$ künstlich aufgeschütteter humoser Schutt

$10-15 \mathrm{~cm}$ rezenter/subrezenter $\mathrm{A}_{\mathrm{h}}$-Horizont einer Braunerde in sandigem Substrat

$10-15 \mathrm{~cm}$ Grauer Übergangshorizont, eisenverarmt?

$30-40 \mathrm{~cm}$ z. T. brauner $\mathrm{B}_{\mathrm{v}}$-Horizont der rezenten/subrezenten Braunerde, auf lößfarbenem, schwach schluffigem Sand oder Sandsteinschutt entwikkelt, in $5 \mathrm{~m}$ breiten Dellen $80-90 \mathrm{~cm}$ mächtig

$30 \mathrm{~cm}$ 1. fossile humose Schicht, in fast schuttfreiem Sand ausgebildet: $A_{\mathrm{h}}$-Horizont?

$40-45 \mathrm{~cm}$ braungelber Schutt, z. T. kantengerundete Steine, durchziehendes Schuttband im Top, $B_{V}$-Horizont 
Schicht Mächtigkeit Geologisch-bodengeographische Ansprache

6

7

8

10

11
10-15 cm 2. fossile humose $\mathrm{Schicht}$ in schuttreichem, sandigem Substrat ( $\mathrm{A}_{\mathrm{h}}$-Horizont), auskeilend, nur lokal entwickelt

$30 \mathrm{~cm}$ braungelber Sand mit wenigen Schuttstücken, stellenweise schwach geschichtet

10-30 cm 3. fossile humose Schicht (Ah-Horizont), wie der 1. fossile humose Horizont dieses Profiles in wechselnder Mächtigkeit durchziehend, mit wenigen Steinen in sandigem Substrat

$130-150 \mathrm{~cm}$ gelber, rostig-brauner Schutt, teils von auskeilenden Limonitkrusten durchzogen, im unteren Teil mehrere Rostbänder, unterer Teil durchgehend rostig gefärbt (Oxydationshorizont)

$40 \mathrm{~cm}$ grauweißer Sand mit wenigen Holzkohlestïcken, in Rinne grauer, sandiger Ton (wie Schlickmaterial), z. T. Ton, mit kräftig schwarzem, humosem Band (Probe 10)

$30 \mathrm{~cm}$ grauer Sandsteinschutt

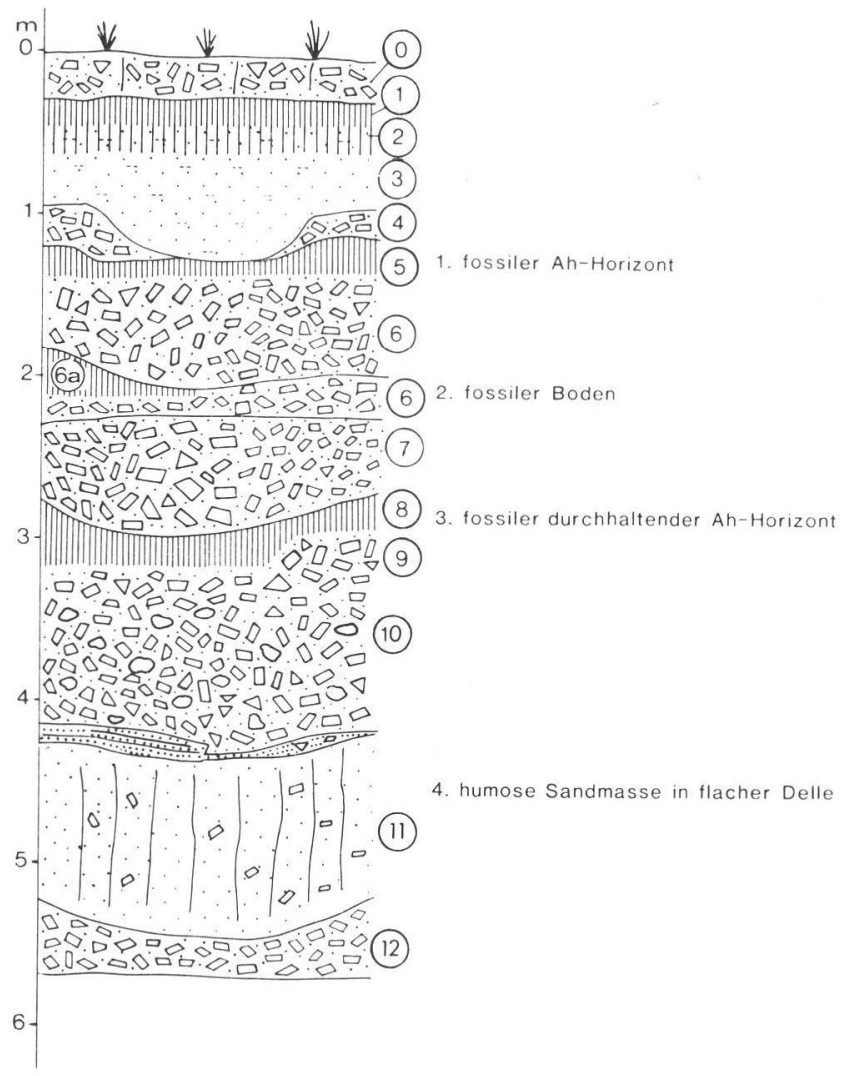

Ab̉b. 4: SW-NE-Wand mit Profil 2; zur Lage vergleiche Abb. 2. Numerierung der Schichten entspricht der Numerierung im Text.

14-C-Alter von Schicht 8: 42800 BP (vgl. Schichtenbeschreibung). 
Tab. 3: Profil 2 (Abb. 4; Tafel 2; Fig. 2), SW-NE-Wand des Profils Cangas de Foz Schicht Mächtigkeit Geologisch-bodengeographische Ansprache

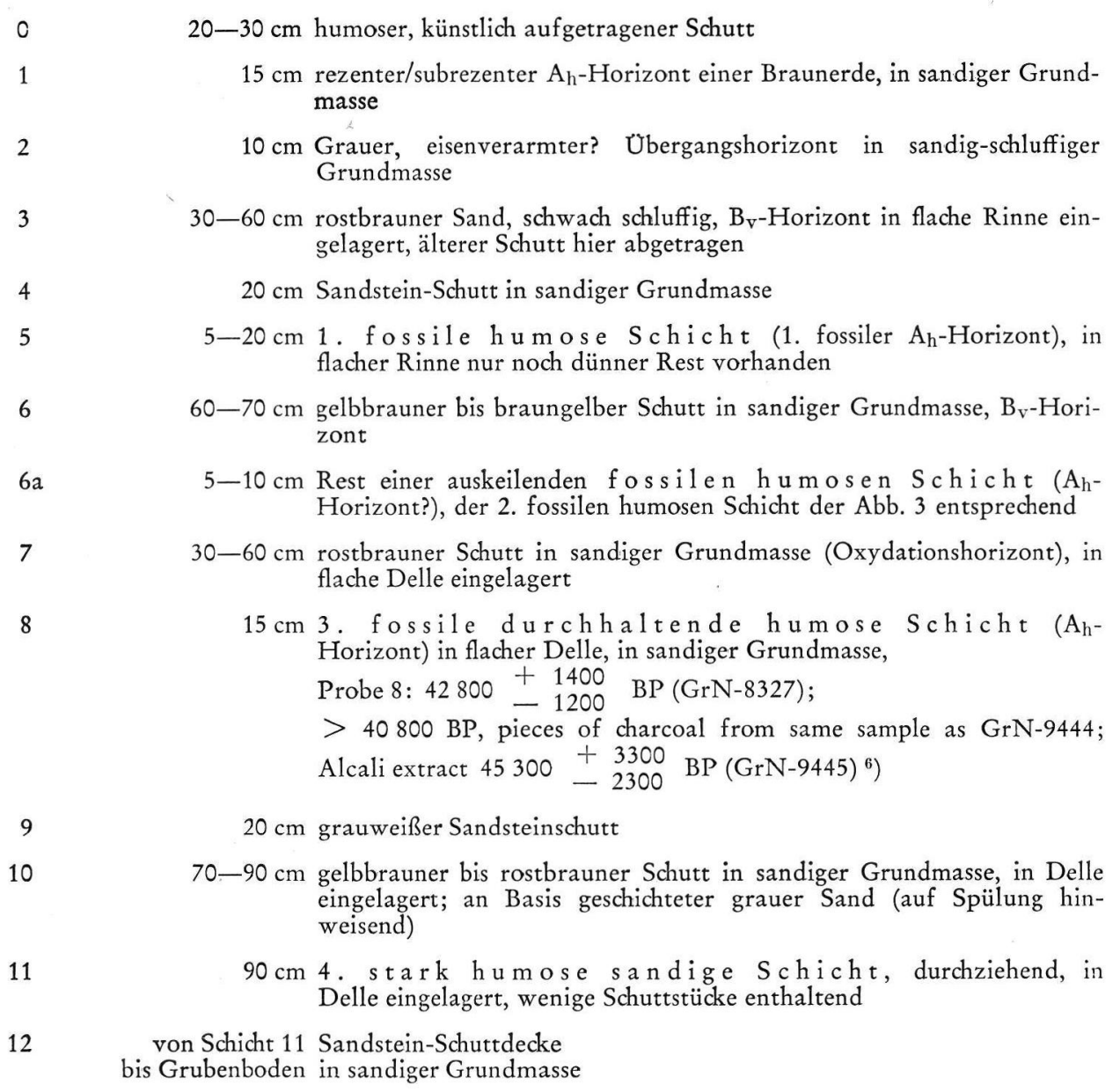

Tab. 4: Profil 3 (Abb. 5), SE-NW-Wand des Profils von Cangas de Foz, der Fig. 3 (Tafel 2) entsprechend

Schicht Mächtigkeit Geologisch-bodengeographische Ansprache

$25 \mathrm{~cm}$ humoser, künstlich aufgeschütteter Schutt
$20 \mathrm{~cm} \begin{aligned} & \text { rezenter/subrezenter } \mathrm{A}_{\mathrm{h}}-\mathrm{H} \text { or i z o n t e in e r B r a u n e r d e in } \\ & \text { sandiger, schwach schluffiger Grundmasse }\end{aligned}$
2
10-15 cm schwach gebleichter Horizont, $z$. T. deutlich, in sandiger Grundmasse

6) Kommentar von Prof. Dr. Mook, Groningen, zu GrN-9445 und GrN-9444: „These three results are comforting. The chances for a disturbing contamination are small." (Brief v. 13. 2. 1980) 


\begin{tabular}{|c|c|c|}
\hline Schicht & Mächtigkeit & Geologisch-bodengraphische Ansprache \\
\hline 3 & $50 \mathrm{~cm}$ & gelbbrauner, steinloser Sand ( $\mathrm{B}_{\mathrm{V}}-$ Horizont $)$ \\
\hline 4 & $50 \mathrm{~cm}$ & gelbbrauner Schutt (Sandstein) in sandiger Grundmasse \\
\hline 5 & $15-30 \mathrm{~cm}$ & $\begin{array}{l}\text { 1. fossile humose sandige } \mathrm{Schicht} \text {, A } \mathrm{A}_{\mathrm{h}} \text {-Horizont, im } \\
\text { oberen Teil grauschwarz, unten schwarz, mit sehr wenigen Steinen, } \\
\text { z. T. auskeilend, z. T. stärker werdend, Probe 5: } 15100 \pm 400 \mathrm{BP} \\
\left.(\mathrm{GrN}-9384) \text {; idem alcali extract } 26900 \pm 200 \mathrm{BP}(\mathrm{GrN}-8326)^{7}\right)\end{array}$ \\
\hline 6 & $50-60 \mathrm{~cm}$ & grauweißer, z. T. gelblich-weißer Schutt in sandiger Grundmasse \\
\hline 7 & $30-35 \mathrm{~cm} 2$ & $\begin{array}{l}\text { 2. fossile humose sandige } \mathrm{Schicht} \text { (2. fossiler } \mathrm{A}_{\mathrm{h}} \\
\text { Horizont), im oberen Teil grau-schmutzig-humos, schwarz-grau im } \\
\text { unteren Teil, wenige Schuttstücke enthaltend, mit wenigen Holzkohle- } \\
\text { stücken, dem Horizont } 8 \text { in Profil } 2 \text { entsprechend }\end{array}$ \\
\hline $8 a$ & $40 \mathrm{~cm}$ & $\begin{array}{l}\text { grau-weißer Schutt in sandiger Grundmasse, fossiler eisenverarmter? } \\
\text { Horizont }\end{array}$ \\
\hline $8 \mathrm{~b}$ & $45 \mathrm{~cm} 1$ & brauner Schutt in sandiger Grundmasse, $\mathrm{B}_{\mathrm{v}}$-Horizont? \\
\hline $8 \mathrm{c}$ & $45-60 \mathrm{~cm}$ & $\begin{array}{l}\text { rostig-brauner Schutt in sandiger Grundmasse, Farbe sehr stark leuch- } \\
\text { tend }\end{array}$ \\
\hline 9 & $30-50 \mathrm{~cm}$ & $\begin{array}{l}\text { bis zum Grubenboden: } s c h w a r z e s, \text { f e uchtes, humoses, } \\
\text { steinloses sandiges Material, Probe } 9\end{array}$ \\
\hline
\end{tabular}

\subsection{Deutung und Datierung der Profile}

Zunächst läßt sich schließen, daß eine wenigstens viermalige Abfolge von sandigen Sedimenten und Schuttdecken abgelagert worden ist, wobei die Schuttdecken hangab in Richtung auf die Küste bewegt wurden. Das ist besonders deutlich in Tafel 2, Fig. 3 erkennbar. Es müssen sich also Zeiten kräftiger Schuttaufbereitung und -verlagerung mit Zeiten der Sandablagerung abgelöst haben. Dies läßt sich m. E. durch die Annahme eines Alternierens von Zeiten kräftiger eiszeitlicher Frostsprengung und Schuttverlagerung (wahrscheinlich in einer kalt-feuchten Phase) und von Sandbildung und Sandverlagerung (wahrscheinlich in einer trocken-kalten Phase) erklären. Dabei halte ich ein zeitweiliges Überwiegen von Sandverwehungen durchaus für möglich, denn in den homogenen lößfarbigen Sandmassen lassen sich weder Steine noch Anzeichen für fluviale Schichtung beobachten.

Zeitweilig muß allerdings Feinmaterial- und Schuttverlagerung synchron verlaufen sein - eine Beobachtung, für die sich auch im lößbedeckten Teil der mitteleuropäischen Mittelgebirge in Hangprofilen immer wieder Indizien finden lassen (vgl. z. B. Poser 1947, 1948; Büdel 1950, 1977; Semmel 1964, 1968). In solchen Profilen findet man bekanntlich in den wenig mächtigen Schuttdecken-Normalprofilen über dem anstehenden Gestein zunächst einen lößfreien Solifluktionsschutt, darüber einen Solifluktionsschutt mit Lößbeteiligung und darüber einen schuttfreien Löß mit der holozänen Bodenbildung.

7) Prof. Dr. Mook, Groningen, schreibt im Brief vom 14. 12. 1979 mit Bezug auf die Probe 5 von Cangas de Foz: „Some alcali extracts which are supposed to contain a possible contamination are still being treated. The higher the age of the extracts, the less chance of a disturbing contamination still remaining in the sample actually dated, the more reliable the final ages. It is surprising that the one extract shows a higher age than the residue." (Damit ist der Alcali-Extrakt von Probe 5 gemeint - Zusatz v. Verf.). 


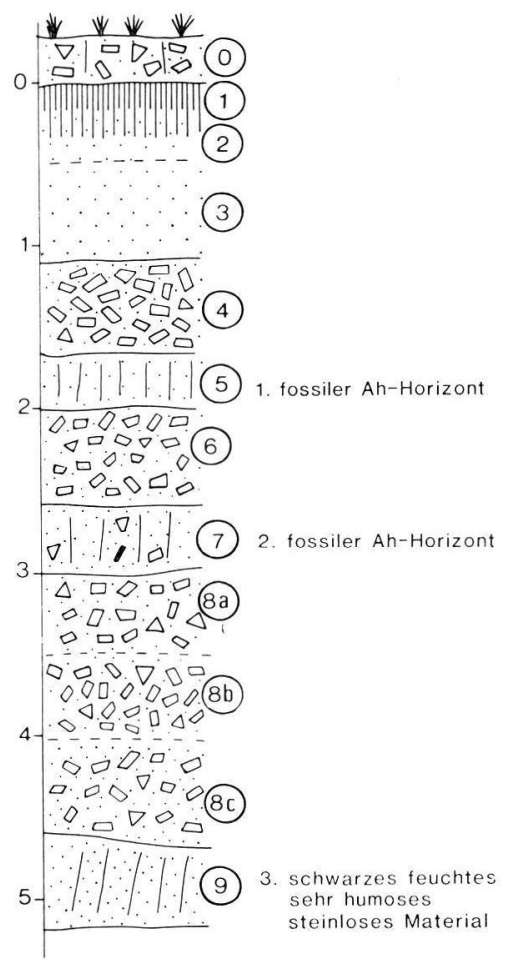

Abb. 5: SE-NW-Wand, Profil 3; zur Lage vgl. Abb. 2.

Numerierung wie im Text. 14-C-Alter für Schicht 5: 15100 BP (vgl. Text.).

Die Schuttdecken bei Cangas de Foz auf den z. T. nur $3^{\circ}$, z. T. $6^{\circ}-8^{\circ}$ geneigten Hängen wurden wahrscheinlich durch eiszeitliches Bodenfließen und durch fluviale Prozesse bewegt. Abspülung läßt sich durch zwei im Aufschluß rechtwinklig angeschnittene flache Dellen, deren Sedimentfüllung z. T. geschichtet ist, belegen. Die Schuttdecken haben bei nur $3^{\circ}$ Neigung und bei der sandigen Matrix kaum günstige Bedingungen für ein Bodenfließen gefunden. Sie weisen nur teilweise eine Einregelung der Längsachsen der Gesteine in Richtung des Gefälles auf, wie sie für Solifluktionsschuttdecken typisch ist. Das heißt, es muß in erster Linie an ein fluvial-solifluidales Faktorengefüge gedacht werden, in dem kräftige Abflüsse in der Lage waren, an der Verlagerung des Schuttes mitzuwirken.

Zur Bodengeographie läßt sich herausstellen, daß hier Übergangsformen von Braunerden zu Podsol-Braunerden als rezente Oberflächenböden zur Ausbildung gelangen, wenn mit Sand durchsetzte Schuttmassen an die Oberfläche treten. Dieselbe Aussage läßt sich auch für weite Aufschlußbereiche in Bezug auf die fossilen Böden machen: auch hier folgen unter kräftigen $A_{h}$-Horizonten dünne, mehr oder weniger graue, wahrscheinlich eisenverarmte Horizonte und darunter kräftig gelbbraune, braune oder rostigbraune BHorizonte.

Tritt dagegen unter dem weitflächig verbreiteten, durch menschliche Tätigkeit verlagerten Schutt im Top des Aufschlusses (Schicht $O$ in Abb. 3-5) ein lößfarbener, schwach schluffhaltiger Sand an die Oberfläche, so ist auch auf diesem ein Boden entwickelt, der als Braunerde angesprochen werden muß. 
Zwei kräftige fossile humose Schichten sind so horizontbeständig und kräftig, daß sie m. E. nur als fossile $A_{h}$-Horizonte fossiler Böden (Braunerden) bzw. Podsol-Braunerden angesehen werden können.

Der oberste fossile $A_{h}$-Horizont, von dem ca. $3 \mathrm{~kg}$ humoses Material für eine ${ }^{14} \mathrm{C}$ Datierung entnommen wurde, ergab ein ${ }^{14} \mathrm{C}$-Alter von $15100 \pm 400 \mathrm{BP}$ (GrN-9384) bzw. idem alcali extract $\left.26900 \pm 200 \mathrm{BP}(\mathrm{GrN}-8326){ }^{8}\right)$ Wegen der großen Differenz dieser beiden ${ }^{14} \mathrm{C}$-Alter kann nur gesagt werden, daß die fossile Bodenbildung mit einiger Sicherheit bereits in die letzte Kaltzeit gehört und daß für ihre Bildung das BöllingInterstadial oder ein älteres Interstadial in Frage kommt.

Das ${ }^{14} \mathrm{C}$-Alter für die zweite kräftige durchziehende humose Schicht (Schicht 8 in Abb. 4), die von der ersten kräftigen fossilen humosen Schicht (Schicht 5 in Abb. 4) noch stellenweise durch eine dünnere, auskeilende humose Schicht (Schicht 6a in Abb. 4) getrennt wird, lautet: $42800+1400 \mathrm{BP}$ (GrN-8327) bzw. $>40800 \mathrm{BP}$; pieces of charcoal from same sample as GrN-8327; Alcali extract $45300+3300$ BP (GrN-9445).

Prof. Mooks Kommentar vom 13. 2. 1980 lautet zu diesen drei Proben: „These three results are comforting. The chances for a disturbing contamination are small." Die untere durchziehende humose Schicht dürfte nach diesen ${ }^{14} \mathrm{C}-\mathrm{Daten}$ am ehesten in das Moershoofd-Interstadial zu stellen sein, vielleicht auch ins Hengelo-Interstadial. Die in den humosen Sanden ebenfalls auftretende Holzkohle, die ein ${ }^{14} \mathrm{C}$-Alter von $>40800$ BP aufweist, zeigt, daß zu dieser Zeit eine holzige Vegetation in der Nähe gewachsen ist. Hiermit liegt eine Parallele zu Untersuchungsergebnissen an der westgalizischen Küste bei La Guardia und Mougas vor (Brosche 1982).

Die in diesem Aufschluß ermittelten ${ }^{14} \mathrm{C}$-Daten lassen erkennen, daß die gesamte Sedimentserie bei Cangas de Foz einen großen Teil des Würmglazials abdeckt und daß in den Interstadialen offenbar so günstige Klimaverhältnisse geherrscht haben, daß eine Bodenbildung und $\mathrm{z}$. T. auch stärkere pflanzliche Produktion stattfinden konnte.

Dagegen ist die Deutung des untersten, stark humosen Bandes im SW-Teil des Aufschlusses (Fig. 1-3, Tafel 2) direkt über dem Grubenboden noch problematisch. Es handelt sich hierbei um tiefschwarze, z. T. muddeartige humose, z. T. mineralische Sedimente, wie sie an sehr feuchten Standorten in Dellen und Depressionen des Reliefs vorzukommen pflegen, nicht dagegen um einen $A_{h}$-Horizont eines fossilen Bodens. Diese schwarzen humosen Sedimente sind in dem Profil von Cangas de Foz auch nur an die Tiefenlinie einer deutlich ausgebildeten flachen Hangmulde (Fig. 2 u. 3, Tafel 2) gebunden. Auch ein dazugehöriger Unterboden ließ sich zu den Sedimenten nicht auffinden.

Stellenweise sind zwischen die m. E. autochthonen kräftigen $\mathrm{A}_{\mathrm{h}}$-Horizonte der rezenten Braunerde und der fossilen Braunerden 1 und 2 weitere schwächere humose Bänder eingeschaltet, die sich jeweils über viele Meter an der Aufschlußwand verfolgen lassen (s. o.). Auf den Figuren sind diese Horizonte kaum sichtbar. Sie ließen sich zwischen dem holozänen Oberflächenboden und dem 1. fossilen Boden sowie zwischen diesem und dem 2. fossilen Boden finden. Diese werden in erster Linie als umgelagertes humoses Bodenmaterial gedeutet, das seinen Ursprung in den kräftig entwickelten autochthonen $A_{h^{-}}$ Horizonten hangauf haben kann. Auf eigenständige Bodenbildungsphasen läßt sich mit inrer Hilfe deshalb nicht schließen, weil sich keine weitere Boden-Profilentwicklung erkennen läßt.

8) Vgl. die Anmerkung 7 auf S. 108. 
Bei der Detailbeschreibung der Abb. 5 (Profil 3, Fig. 3, Tafel 2) wurde bereits darauf hingewiesen, daß die zwei horizontbeständigen, kräftigen, m. E. autochthonen $A_{h}-$ Horizonte an der von SE nach NW ziehenden Wand sehr dunkel (z. T. schwarzgrau oder schwarz) sind, während die sandigen Sedimente über ihnen eine schmutzig-graue, graue oder dunkelgraue Farbe aufweisen. Dieses Phänomen bedarf einer weiteren detaillierten Untersuchung. Als wahrscheinliche Erklärung kann gelten, daß nach Ausbildung der autochthonen $\mathrm{A}_{\mathrm{h}}$-Horizonte in der folgenden Aktivitätsphase sandiges, schwach humoses Material, das hangabwärts bzw. dellenabwärts verlagert wurde, auf den $A_{h}$-Horizont aufgespült wurde.

Ebenfalls problematisch bleiben die rostig-braunen Farben einiger Schuttpartien, die rostigen Oxydationsbänder und die Limonitkrusten. Es ist wenig wahrscheinlich, daß die Oxydationsvorgänge in einer Zeit wirkten, als der Meeresspiegel wesentlich höher lag als heute, da die ${ }^{14} \mathrm{C}$-Daten für eine würmzeitliche Schichtenfolge sprechen. Wahrscheinlicher ist, daß ein zeitweilig (vielleicht nur jahreszeitlich) existierender Frostboden das Versickern des Infiltrationswassers verhinderte und es zu einem oberflächenparallelen Abfluß zwang. Um einen echten, über längere Zeiträume persistenten, tiefgreifenden Dauerfrostboden wird es sich dabei nicht gehandelt haben; denn bisher fehlen für die Ableitung seiner ehemaligen Existenz hier (und übrigens auf der gesamten Iberischen Halbinsel in den tieferen Lagen) die typischen Kennformen (fossile Eiskeilpseudomorphosen), wie zuletzt bei Brosche (1980) dargelegt wurde. Südlich dieser Lokalität fand aber Nonn (1966:86, 96, Planche II und Fig. 18) bei Guitirriz an der Nationalstraße La Coruña-Madrid (km-Stein 554,5) einen recht deutlich entwickelten doppelten Kryoturbationshorizont, der durchaus die Annahme der zeitweiligen Existenz eines dünnen Frostbodens, der mehrere Jahre nicht abtaute, zuläßt.

\section{Schriftenverzeichnis}

Brosche, K.-U. (1978): Beiträge zum rezenten und vorzeitlichen periglazialen Formenschatz auf der Iberischen Halbinsel. - Abh. d. Geogr. Inst. d. FU Berlin, Sonderhefte, 1: 287 S.; Berlin.

-. (1980): Zum Problem echter und unechter Periglazialerscheinungen im Ebrobecken und im Gebiet südlich von Madrid. - Beiträge zur Geomorphologie und Länderkunde, Hrsg. B. Hofmeister und A. Steinecke, Berliner Geogr. Studien, 7: 289-307; Berlin.

- (1982): Studien zu jungpleistozänen und holozänen Sedimenten und fossilen Böden im Küstengebiet von West-Galizien (NW-Spanien). - Eiszeitalter u. Gegenwart, 32: 63-80; Hannover.

- \& WALther, M. (1977): Geomorphologische und bodengeographische Analyse holozäner, jung- und mittelpleistozäner Sedimente und Böden in Spanien und Südfrankreich. - Catena, 3: 311-342; Gießen.

-, _ \& - (1980): Lößprofile von Vaake (B1. 4523 Münden) und Albungen (Bl. 4725 Bad Sooden-Allendorf) in Nordhessen. - Geol. Jb. Hessen, 108: 143-150; Wiesbaden.

Brunnacker, K. (1957): Die Geschichte der Böden im jüngeren Pleistozän in Bayern. - Geologica Bavarica, 34: 1-95; München.

— \& LožEK, V. (1969): Löß-Vorkommen in Südostspanien. - Z. f. Geomorph., N. F., 13: 297316; Berlin. 
BüDEL, (1950): Die Klimaphasen der Würmeiszeit. - Die Naturwissenschaften, 37: 438-449; Berlin, Göttingen, Heidelberg.

- (1977): Klima-Geomorphologie. 304 S.; Berlin - Stuttgart.

FINK, J. (1956): Zur Korrelation der Terrassen und Lösse in Ơsterreich. — Eiszeitalter u. Gegenwart, 7: 49-77; Ơhringen.

Franz, H. (1967): Beiträge zur Kenntnis der Bodenentwicklung in NW-Spanien auf Grund fossiler Böden. - Annales de Edafologia y Agrobiologia, 26: 33-51; Madrid.

Hernández Pachéco, F., Llopís Lladó, N., Jorda Cerda, F. \& Martinez, J. A. (1957): El Cuaternario de la Region Cantabrica. V. Vongrès International INQUA. Libro Guia de la Excursion N2; Madrid - Barcelona.

LiEBERoth, I. (1959): Beobachtungen im nordsächsischen Lößgebiet. - Z. Pflanzenernährung, Düngung, Bodenkunde, 87 (132): 97-108; Weinheim.

- (1963): Lößsedimentation und Bodenbildung während des Pleistozäns in Sachsen. - Geologie, 12: 149-187; Berlin.

Llopís Lladó, N. (1955): Las depósitos de la costa cantabrica entre los Cabos Bustio y Vidio. Speleón, 6: 333-347; Oviedo.

- (1957): La plataforma costera de la costa asturiana entre Cabo Busto y el Eo y sus depósitos. - V. Congrès International INQUA. Resumés de Communications, p. 112; Madrid - Barcelona.

- \& JoRda, F. (1957): Mapa del Cuaternario de Asturias. Oviedo.

Nonn, H. (1960): Les dépots de la rasa cantabrique dans la partie occidentale (Galice; Espagne) - Rev. de Géomorphologie Dynamique, 7-8-9: 97-105; Strasbourg - Paris.

- (1966): Les régions cotières de la Galice (Espagne). Etude géomorphologique. — Fondation Baulig, III: 591 S.; Strasbourg.

- (1969): Evolution Géomorphologique et Types de Relief en Galice Occidentale et Septentrionale. - Rev. de Géogr. Physique et de Géologie Dynamique (2), XI, 1:31-50; Paris.

Poser, H. (1947): Auftautiefe und Frostzerrung im Boden Mitteleuropas während der WürmEiszeit. - Die Naturwissenschaften, 34: 232-238 und 262-267; Berlin, Göttingen, Heidelberg.

- (1948): Boden- und Klimaverhältnisse in Mittel- und Westeuropa während der WürmEiszeit. - Erdkunde, 2: 53-68; Bonn.

-- \& HövermanN, J. (1951): Untersuchungen zur pleistozänen Harzvergletscherung. - Abh. d. Braunschweig. Wiss. Ges., 3: 61-115; Braunschweig.

Rohdenburg, H. (1968): Jungpleistozäne Hangformung in Mitteleuropa - Beiträge zur Kenntnis, Deutung und Bedeutung ihrer räumlichen und zeitlichen Differenzierung. - Göttinger Bodenkundl. Ber., 6: 3-107; Göttingen.

- (1971): Einführung in die klimagenetische Geomorphologie. Gießen.

- \& SABELBerg, U. (1969a): „Kalkkrusten“ und ihr klimatischer Aussagewert - Neue Beobachtungen aus Spanien und Nordafrika. - Göttinger Bodenkundl. Ber., 7: 3-26; Göttingen.

-, _ \& - (1969b): Zur landschaftsökologisch-bodengeographischen und klimagenetisch-geomorphologischen Stellung des westlichen Mediterrangebietes. - Göttinger Bodenkundl. Ber., 7: 27-47; Göttingen.

- , - \& - (1973): Quartäre Klimazyklen im westlichen Mediterrangebiet und ihre Auswirkungen auf die Relief- und Bodenentwicklung. - Catena, 1; Gießen.

Ruske, R. \& WüNSChe, M. (1961): Lösse und fossile Böden im mittleren Saale- und Unstruttal. Geologie, 10: 9-29; Berlin.

Rutte, E. (1960): Kalkkrusten in Spanien. - Neues Jahrbuch f. Geol. u. Pal., Abh., 106: 52-138.

Schönhals, E., Rohdenburg, H. \& Semmel, A. (1964): Ergebnisse neuerer Untersuchungen zur Würmlößgliederung in Hessen. - Eiszeitalter u. Gegenwart, 15: 199-206; Ơhringen. 
SEMmel, A. (1964): Junge Schuttdecken in hessischen Mittelgebirgen. - Notitzblatt d. Hess. Landesamtes f. Bodenforschg., 92: 275-285; Wiesbaden.

- (1968): Studien über den Verlauf jungpleistozäner Formung in Hessen. - Frankfurter Geogr. Hefte, 45: 133 S.; Frankfurt.

- (1974): Der Stand der Eiszeit-Forschung im Rhein-Main-Gebiet. - Rhein-Mainische Forschungen, 78: 9-56; Frankfurt a. M.

Tricart, J. (1966): Quelques aspects des phénomènes périglaciaires quaternaires dans la Péninsule Ibérique. - Biuletyn Periglacjalny, 14: 313-327; Lødz.

ÜNGER, G. \& RAU, D. (1964): Lößexkursion Thüringen. Subkommission für Lößstratigraphie der INQUA. Berlin 1964: 7-25; Berlin.

Manuskript eingegangen am 4. 3. 1983 
Fig. 1: Oberer Teil des Küstenprofils von der Playa de Barañán bei Campo Branqueza (westl. $\mathrm{La}$ Coruña). Unter den rezenten bis subrezenten Flugsanden (Schicht Nr. 1 in Abb. 1) fallen besonders die humosen Sedimente der 1. und 2. fossilen Bodenbildung auf. Dezimetereinteilung auf $1 \mathrm{~m}$ langer Meßlatte.

Fig. 2: Küstensedimente an der Playa de Barañán. In oberer rechter Bildecke noch die rezenten/ subrezenten Flugsande erkennbar. Die Abfolge von schuttreichen Solifluktionsschuttdecken und von schuttarmen und schuttlosen Schichten wird deutlich. Humose Lagen nicht erkennbar. Meßlatte $1 \mathrm{~m}$ lang.

Fig. 3: Lokalität wie in Fig. 1 und 2. Blick nach W. Natürliches Kliff in den hier in Meernähe konvergierenden und dadurch zusammengedrängten Sedimenten. Hauptmasse ist typische eiszeitliche Solifluktionsschuttdecke. Unten in fast steinfreien, schluffig-sandigen Schichten schwach eine dunklere humose Schicht erkennbar. Dieser Profilabschnitt schließt sich rechts an Sedimente der Fig. 1 an. 

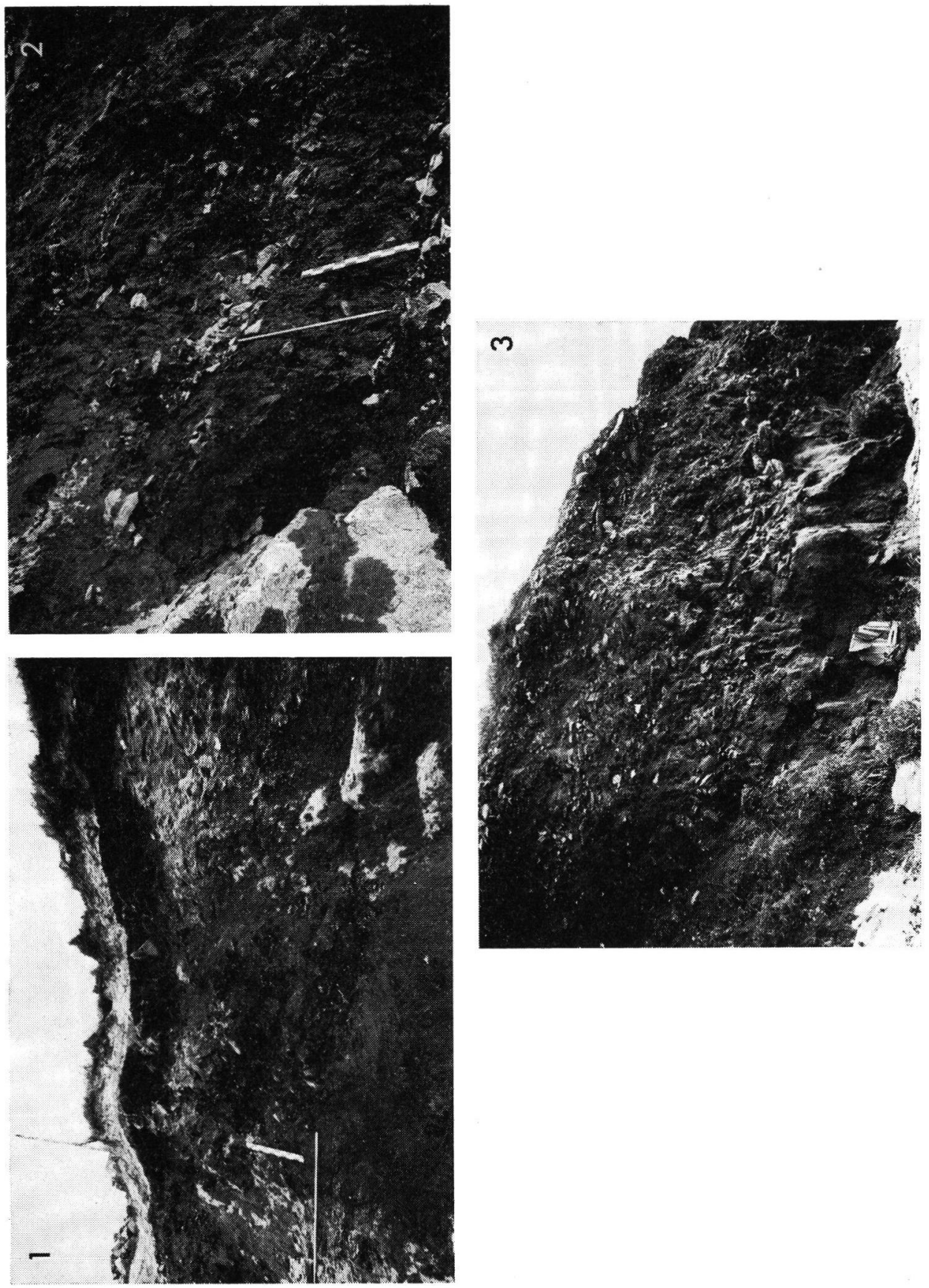
Fig. 1: Úbersichtsfoto des Aufschlusses bei Cangas de Foz am km-Stein 416 der Nationalstraße C 642 . Abb. 3, 4, 5, Fig. 2 und 3 wurden links und rechts vom Baumaterial aufgenommen. Schwache Dellenbildung (vgl. Fig. 2) an linker Wand erkennbar. Abfolge von Sandsteinschuttdecken, sandigschluffigem Feinmaterial und humosen Schichten.

Fig. 2: Lokalität wie in Fig. 1. Detailansicht der Sediment-Boden-Abfolge im Bereich einer flachen Delle. Numerierung der Schichten entspricht der in Abb. 4 (Profil 2). Mächtigkeit der humosen Schichten in Dellentiefenlinien am größten. Schicht 8 ergab ${ }^{14} \mathrm{C}$-Alter von $42800 \mathrm{BP}$ (vgl. Schichtenbeschreibung). Abb. 4 ca. $3 \mathrm{~m}$ links von Meßlatte.

Fig. 3: Lokalität wie in Fig. 1. Abfolge von steinlosen und steinreichen Schichten hier sehr deutlich. Humose Schichten gut erkennbar. Schuttreiches Sediment am Top mit Vegetation künstlich aufgetragen. Meßlatte $1 \mathrm{~m}$ lang. 

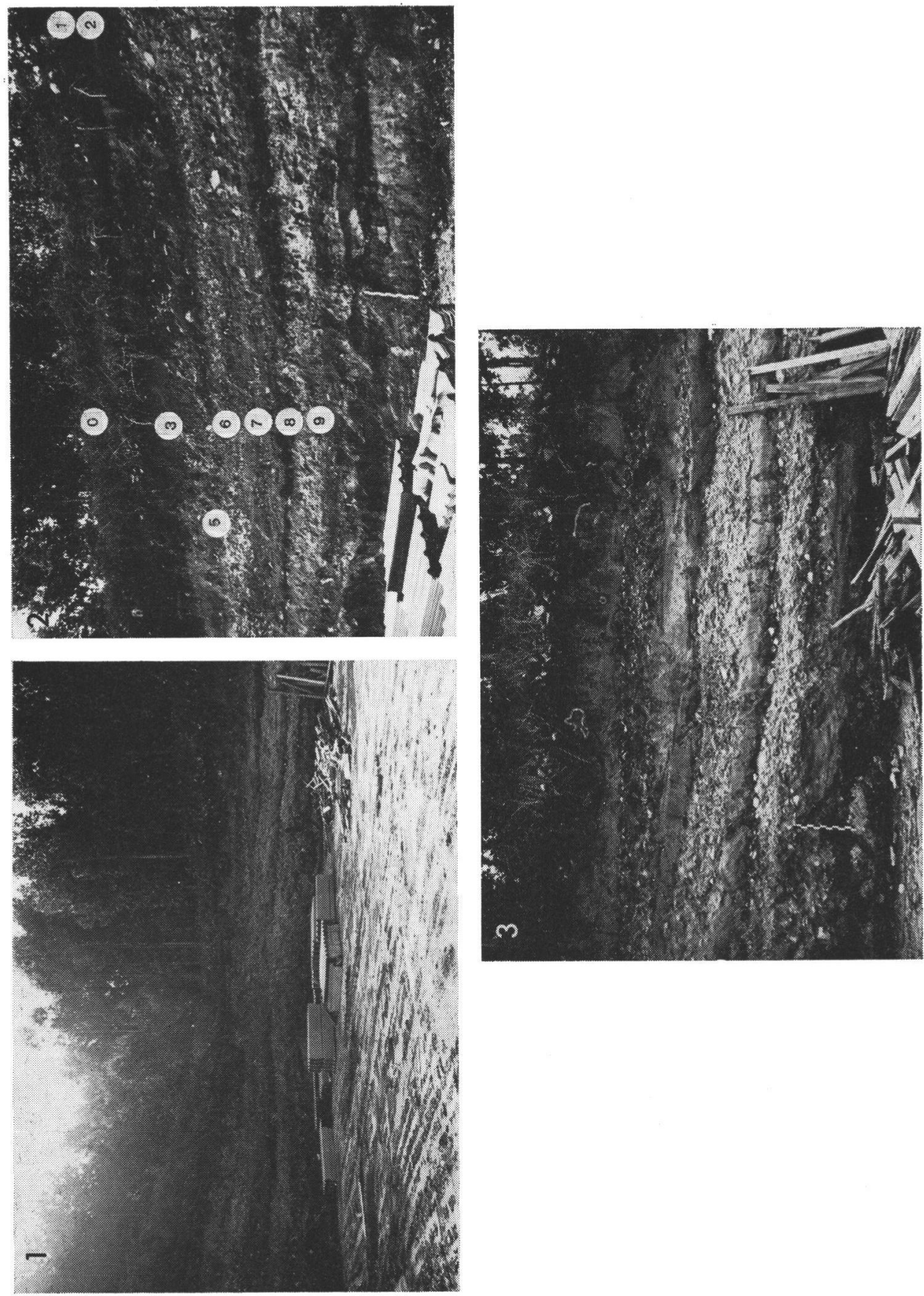
